\title{
CT scanning may adversely influence choice of surgery in paediatric papillary thyroid carcinoma - a note of caution
}

\author{
$S$ Andronikou \\ MB BCh, FCRad (Diag) (SA), FRCR (Lond) \\ Formerly: Department of Paediatric Radiology \\ Red Cross Children's Hospital and \\ University of Cape Town
}

\section{Abstract}

Papillary thyroid carcinoma may be multifocal in up to $50 \%$ of affected children. Surgeons must weigh the higher risks of recurrence when surgical resection is less than subtotal thyroidectomy against the higher risks of complications with subtotal/total thyroidectomy. An 11-year-old girl presented to our surgical department with a thyroid mass. Computed tomography (CT) revealed a lesion, missed by ultrasonography, which reversed the decision to perform conservative thyroid surgery. The lesion proved to be non-neoplastic. CT may be a useful adjunct when ultrasound demonstrates a solitary lesion and conservative surgery is considered, and may prove to be able to distinguish neoplastic from non-neoplastic lesions based on contrast enhancement characteristics, but caution is advised in assuming that all nodules are neoplastic.

\section{Introduction}

The literature presents two views regarding the treatment of paediatric papillary thyroid carcinoma. There are those supporting conservative surgical procedures and those supporting subtotal or total thyroidectomy. ${ }^{1-7}$ Surgeons must weigh the higher risks of recurrence when surgery is less than subtotal thyroidectomy against the higher risks of complications with subtotal/total thyroidectomy. CT may be a useful adjunct when ultrasound demonstrates a solitary lesion and conservative surgery is considered, and may prove to be able to distinguish neoplastic from non-neoplastic lesions based on contrast enhancement characteristics.

\section{Case report}

An 11-year-old girl presented to our surgical department with a thyroid mass. Computed tomography (CT) revealed a finding, missed by ultrasonography, which reversed the decision to perform conservative thyroid surgery. Ultrasound demonstrated a single lesion in the left thyroid lobe (Fig. 1). CT confirmed the primary lesion in the left thyroid lobe measuring $2.2 \times 2.2 \times 3 \mathrm{~cm}$, but also revealed a small right lobe lesion measuring $0.1 \times 0.2 \times 1.5 \mathrm{~cm}$ (Fig. 2a), which was not observed on ultrasound. Contrast CT showed enhancement of the left lobe nodule, but no enhancement of the right lobe nodule (Fig. 2b). More extensive surgery than originally planned was undertaken based on this finding, presuming that the right nodule was also a possible focus of malignancy. Pathological examination diagnosed the larger nodule to be a papillary thyroid carcinoma with vascular invasion but concluded that the smaller nodule was not neoplastic.

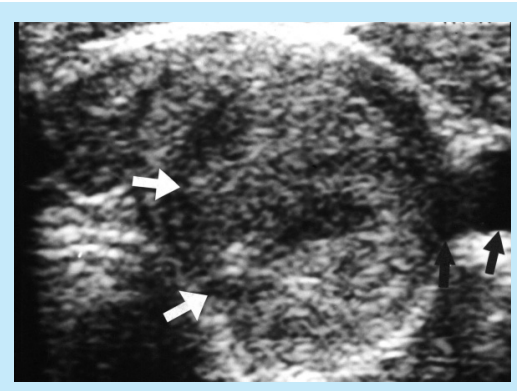

Fig. 1. Ultrasound demonstrates the inhomogeneous left thyroid lobe mass (white arrows) and the adjacent carotid and jugular vessels (black arrows).

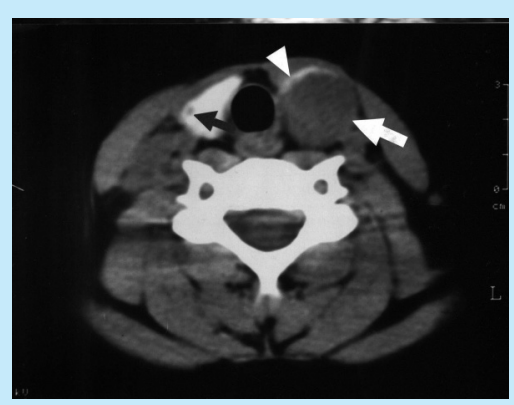

Fig. 2a. Non-contrast CT scan of the neck shows the low-density nodule in the left thyroid lobe (whte arrow) with a thin rim of normal dense thyroid parenchyma anteriorly (white arrowhead). A small second low-density lesion is demonstrated in the right lobe laterally (black arrow).

\section{Discussion}

In the consideration of surgical treatment of paediatric papillary thyroid carcinoma, the higher risks of 


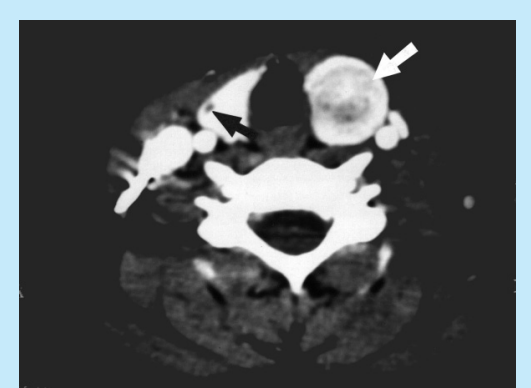

Fig. 2b. Post contrast CT scan of the same region demonstrates inhomogeneous enhancement of the larger nodule (white arrow) making it more difficult to differentiate from normal parenchyma. The small right lobe lesion does not enhance and is now more evident (black arrow).

recurrence when surgery is less than subtotal thyroidectomy must be weighed against the higher risks of complications with subtotal/total thyroidectomy. Complications of subtotal/total thyroidectomy include hypoparathyroidism in up to $30 \%$ of patients and recurrent laryngeal nerve injury in up to $11 \%$ of patients. ${ }^{1-7}$ Papillary thyroid carcinoma may be multifocal in up to $50 \%$ of affected children $^{1-3,5,7}$ and, as a result, recurrence rates are higher when less than subtotal thyroidectomy is performed. ${ }^{2}$ Residual thyroid tissue also diminishes the specificity of thyroglobulin as a tumour marker and interferes with radioiodine as a diagnostic tool postoperatively. ${ }^{3}$ Some authors maintain, however, that there is no evidence that patients who undergo total thyroidectomy fare better than those with conservative surgery at long-term followup, ${ }^{2,3,5}$ even though recurrence in thyroid remnants is higher in children than in adults.

Ultrasonography is used to rule out thyroid anomalies, to determine if nodules are solitary or multiple, and to determine if nodules are cystic or solid. ${ }^{6,8}$ A cystic nodule does not exclude malignancy ${ }^{8}$ and therefore imaging is largely directed toward excluding multifocality. The sensitivity for ultrasound detection of microscopic nodules (less than what size?) is quoted to be $50 \%$. When there is a solitary nodule, fine needle aspiration (FNA) may be sufficient to confirm or exclude malignancy $y^{4,8}$ and conservative surgery can be considered. Scintigraphy in patients who have undergone ultrasonography and FNA is not expected to contribute useful information in such cases and has been omitted in some protocols. ${ }^{8}$ CT scanning has only been recommended for the evaluation of paediatric thyroid lesions by two authors. ${ }^{1,6}$ In our patient, CT proved to be more sensitive than ultrasound in detecting the small right thyroid lesion. Although the small lesion was not neoplastic, it led to more aggressive surgery. With non-contrast CT, these lesions are seen as low-density lesions against the dense background of iodine-rich normal parenchyma (Fig. 2a). Conversely, enhancement of a small lesion may make it less conspicuous on CT compared with the adjacent dense thyroid tissue. Contrast CT in our patient showed enhancement of the primary neoplastic left lobe nodule, but no enhancement of the non-neoplastic right lobe nodule. This may be a means of differentiating neoplastic from non-neoplastic lesions, but this remains to be proved.

\section{Conclusion}

Identification of more than one lesion in childhood thyroid carcino- ma will affect surgical management in centres where conservative surgery is preferred, and imaging must be directed towards identification of these. CT may be a useful adjunct when ultrasound demonstrates a solitary lesion and conservative surgery is considered, and may prove to be able to identify small lesions and possibly distinguish neoplastic from non-neoplastic lesions based on contrast enhancement characteristics. However, prospective studies to compare CT and ultrasound detection of thyroid lesions are difficult to perform because of the rarity of thyroid malignancy in childhood ( $1-1.5 \%$ of all malignancies in this age group). ${ }^{5,7}$

\section{References}

1. La Quaglia MP, Black T, Holcomb GW 3rd, et a Differentiated thyroid carcinoma: Clinical characteristics, treatment and outcome in patients under 21 years of age who present with distant metastases. A report from the surgical discipline committee of the children's cancer group. I Pediatr Surg 2000; 35: 955-960.

2. Welch Dinauer CA, Tuttle RM, Robie DK, Mc Clellan DR, Francis GL. Extensive surgery improves recurrence-free survival for children and young patients with class I papillary thyroid carcinoma. J Pediatr Surg 1999; 34: 1799-1804.

3. Kuefer MU, Moinuddin M, Heideman RL, et al. Papillary thyroid carcinoma: demographics, treatment and outcome in eleven pediatric patients treated at a single institution. Med Pediatr Oncol 1997; 28: 433-440.

4. Karguzel G, Tanyel FC, Buyukpamukcu N, Hicsonmez A. Is there any predictive characteristic for malignancy in thyroid enlargements during childhood? Eur J Pediatr Surg 1996; 6: 70-74.

5. Massimino M, Gasparini M, Ballerini E, Del Bo R. Primary thyroid carcinoma in children: a retrospective study of 20 patients. Med Pediatr Oncol 1995; 24: 13-17.

6. Gorlin JB, Salan SE. Thyroid cancer in childhood. Endocrinol Metab Clin North Am 1990; 19: 649-662.

7. Desjardins JG, Bass J, Leboeuf G, et al. A twenty-year experience with thyroid carcinoma in children. J Pediatr Surg 1988; 23: 709-713.

8. Hung W. Solitary thyroid nodules in 93 children and adolescents: a 35 years experience. Horm Res 1999; 52: 15-18. 